Pacific

Journal of

Mathematics

THE ISOMORPHISM PROBLEM FOR INCIDENCE RINGS

Gene Abrams, Jeremy Haefner, and Angel del Río 


\title{
THE ISOMORPHISM PROBLEM FOR INCIDENCE RINGS
}

\author{
Gene Abrams, Jeremy Haefner, and Angel Del Río
}

Let $P$ and $P^{\prime}$ be finite preordered sets, and let $R$ be a ring for which the number of nonzero summands in a direct decomposition of the regular module $R_{R}$ is bounded. We show that if the incidence rings $I(P, R)$ and $I\left(P^{\prime}, R\right)$ are isomorphic as rings, then $P$ and $P^{\prime}$ are isomorphic as preordered sets. We give a stronger version of this result in case $P$ and $P^{\prime}$ are partially ordered. We show that various natural extensions of these results fail. Specifically, we show that if $\left\{P_{j} \mid j \in \Omega\right\}$ is any collection of (locally finite) preordered sets then there exists a ring $S$ such that the incidence rings $\left\{I\left(P_{j}, S\right) \mid j \in \Omega\right\}$ are pairwise isomorphic. Additionally, we verify that there exists a finite dimensional algebra $R$ and locally finite, nonisomorphic partially ordered sets $P$ and $P^{\prime}$ for which $I(P, R) \simeq I\left(P^{\prime}, R\right)$.

Throughout this article $P$ will denote a locally finite preordered set; so $P$ is a set equipped with a reflexive, transitive relation such that for any two elements $x, y \in P$, the set $\{z \in P \mid x \leq z \leq y\}$ is finite. $R$ will denote an associative unital ring. The incidence ring of $P$ with coefficients in $R$, denoted by $I(P, R)$ (or simply by $A$ throughout this article), is the ring of functions $\{f: P \times P \rightarrow R \mid f(x, y)=0 \forall x \not \leq y\}$; multiplication is given by $(f g)(x, y)=\sum_{x \leq z \leq y} f(x, z) g(z, y)$ (and is well-defined due to the local finiteness condition on $P$ ). The ring $I(P, R)$ may also be viewed as square matrices with entries in $R$, whose rows and columns are indexed by $P$, for which the $(x, y)$ coordinate is 0 whenever $x \not \leq y$.

Given locally finite preordered sets $P$ and $P^{\prime}$, and rings $R$ and $R^{\prime}$, the Isomorphism Problem for Incidence Rings is posed as follows: If the incidence rings $I(P, R)$ and $I\left(P^{\prime}, R^{\prime}\right)$ are isomorphic, under what hypotheses may we conclude that $P \simeq P^{\prime}$ as preordered sets and/or $R \simeq R^{\prime}$ as rings? Our investigation in this article focuses on a specific version of the Isomorphism Problem; namely, we find various conditions on $P, P^{\prime}$, and $R$ for which the hypothesis $I(P, R) \simeq I\left(P^{\prime}, R\right)$ yields the conclusion that $P \simeq P^{\prime}$ as preordered sets.

A number of authors have investigated this version of the Isomorphism Problem. An outstanding historical sketch and bibliography related to the Problem is presented in [3, Section 7.2]. For our purposes, two articles 
in this area deserve explicit mention. In [4, Theorem 4.3], Voss gives an affirmative answer to the Isomorphism Problem in the case where $P, P^{\prime}$ are locally finite preordered sets, and $R$ is an indecomposable semiperfect ring. In fact, in a parenthetical remark made near the end of [4], Voss actually extends his solution to coefficient rings which are finite direct sums of indecomposable semiperfect rings, each of whose associated preordered sets is pairwise isomorphic. More recently, in [1, Theorem 2.4], Dăscălescu and Van Wyk solve the Isomorphism Problem in the situation where $P, P^{\prime}$ are finite preordered sets, and $R$ is semiprime noetherian. In both of these articles, various examples are given to show that the hypotheses used cannot be eliminated.

In this article we make two types of contributions to the investigation of the Isomorphism Problem. In Section 1 we extend the aforementioned results in the situation where $P$ and $P^{\prime}$ are finite. We do so by establishing the following solutions to two versions of the Isomorphism Problem.

Solution 1 (cf. Theorem 1.12). Let $R$ be a ring with the property that the integer $\max \left(\left\{n \mid\right.\right.$ there exist nonzero right ideals $K_{1}, K_{2}, \ldots, K_{n}$ with $R=$ $\left.\left.K_{1} \oplus K_{2} \oplus \cdots \oplus K_{n}\right\}\right)$ exists, and let $P$ and $P^{\prime}$ be finite preordered sets. If $I(P, R) \simeq I\left(P^{\prime}, R\right)$ as rings, then $P \simeq P^{\prime}$ as preordered sets.

Numerous classes of rings have the property mentioned in Solution 1; we say such rings have finite summand length. (It is not hard to show that this property is left/right symmetric.) Such classes include rings with Goldie dimension (e.g. Noetherian rings), and semiperfect rings (e.g. finite dimensional algebras). In particular, Solution 1 extends [1, Theorem 2.4], even more so than is needed to answer in the affirmative the conjecture made at the end of that article. Along the way to developing the tools needed to prove Theorem 1.12, we establish:

Solution 2 (cf. Theorem 1.6). Let $R$ be a ring with the property that there are only finitely many direct summands of the right regular module $R_{R}$ which are in addition two-sided ideals of $R$, and let $P$ and $P^{\prime}$ be finite partially ordered sets. If $I(P, R) \simeq I\left(P^{\prime}, R\right)$ as rings, then $P \simeq P^{\prime}$ as partially ordered sets.

Our method of attack used in establishing these two solutions is somewhat similar in flavor to that used by Voss [4]. As we do not necessarily work over semiperfect coefficient rings, we are not afforded the luxury of working with a canonical set of indecomposable projective modules (which proved so helpful to Voss and others). Instead, we focus on a particular set of projective right ideals $D(T)$ for a general ring $T$, and then show that there is a strong relationship between $D(R)$ and $D(I(P, R))$. This relationship allows us to produce an isomorphism between the direct products of certain preordered (resp. partially ordered) sets, which then puts us in position 
to use a 'cancellation' theorem of Lovasz [2, Theorem 4.3] to establish the desired result.

Our second contribution surfaces in Section 2, where we show that natural extensions of various solutions to the Isomorphism Problem (our own, as well the solutions of others mentioned above) fail. We show first (Proposition $2.1)$ that Solution 2 cannot be extended to preordered sets. Next we show (Proposition 2.3) that if $\left\{P_{j} \mid j \in \Omega\right\}$ is any collection of locally finite preoredered sets then there exists a ring $S$ such that the incidence rings $\left\{I\left(P_{j}, S\right) \mid j \in \Omega\right\}$ are pairwise isomorphic, thereby demonstrating that any solution to the Isomorphism Problem must contain some stipulation on the structure of the coefficient rings. Finally, we answer a question posed at the end of [4] by demonstrating (Proposition 2.4) the existence of a finite dimensional algebra $R$ and locally finite, nonisomorphic partially ordered sets $P$ and $P^{\prime}$ for which $I(P, R) \simeq I\left(P^{\prime}, R\right)$.

\section{Solutions to the Isomorphism Problem.}

We begin with some notation and observations. For a $\operatorname{ring} T$ we let $\operatorname{Id}(T)$ denote the set of two-sided ideals of $T$, while $\operatorname{Id}\left(T_{T}\right)$ denotes the set of right ideals of $T$. We let

$$
\begin{aligned}
D(T) & =\left\{K \in \operatorname{Id}(T) \mid K \text { is a direct summand of } T_{T}\right\} \\
& =\left\{e T \mid e=e^{2} \in T \text { and } T e \subseteq e T\right\} .
\end{aligned}
$$

In the sequel we will often view $D(T)$ as a partially ordered set with relation $\subseteq$. It is not hard to show that $D(T)$ is closed under finite sums. For two preordered sets $P$ and $P^{\prime}$ with $x \in P$ we let

$$
\hat{x}=\{y \in P \mid x \leq y \leq x\}
$$

$$
\hat{P}=\{\hat{y} \mid y \in P\} \quad \text { (the partially ordered set associated to } P \text { ) }
$$

$\operatorname{AntiHom}\left(P, P^{\prime}\right)=$ the set of preordered anti-homomorphisms

$$
\text { from } P \text { to } P^{\prime} \text {. }
$$

The set $\operatorname{AntiHom}\left(P, P^{\prime}\right)$ is preordered, where for $F, G \in \operatorname{AntiHom}\left(P, P^{\prime}\right)$ we set $F \leq G$ in case $F(x) \leq G(x)$ for all $x \in P$. The preordered sets $P$ and $P^{\prime}$ are isomorphic in case there exists a set bijection $H: P \rightarrow P^{\prime}$ such that both $H$ and $H^{-1}$ preserve the respective orders on $P$ and $P^{\prime}$. If $|\hat{x}|=n$, then for any $\operatorname{ring} T$ we have $I(\hat{x}, T) \simeq M_{n}(T)$, the ring of $n \times n$ matrices with coefficients in $T$. For a preordered set $P$ and a ring $R$ we set $A=I(P, R)$. If $X$ is a subset of $P, e_{X}$ denotes the idempotent of $A$ given by $e_{X}(x, y)=1$ if $x=y \in X$, and $e_{X}(x, y)=0$ otherwise. For $x \in P$ we denote $e_{\{x\}}$ by $e_{x}$. 
Our first task is to identify the set of two-sided ideals of $A=I(P, R)$ in case $P$ is finite. Given a map $\Psi: P \times P \rightarrow \operatorname{Id}\left(R_{R}\right)$, we let

$$
I(\Psi)=\{\alpha \in A \mid \alpha(x, y) \in \Psi(x, y) \text { for all } x \leq y \text { in } P\} .
$$

Lemma 1.1. Let $P$ be a preordered set, and let $\Psi: P \times P \rightarrow \operatorname{Id}\left(R_{R}\right)$ be a map.

(1) $I(\Psi) \in \operatorname{Id}\left(A_{A}\right)$ if and only if $\Psi(x, y) \subseteq \Psi\left(x, y^{\prime}\right)$ for every $x \leq y \leq y^{\prime}$ in $P$.

(2) $I(\Psi) \in \operatorname{Id}(A)$ if and only if $\Psi(x, y) \in \operatorname{Id}(R)$ and $\Psi(x, y) \subseteq \Psi\left(x^{\prime}, y^{\prime}\right)$ for every $x^{\prime} \leq x \leq y \leq y^{\prime}$ in $P$.

(3) If $P$ is finite, then every two-sided ideal of $A$ is of the form $I(\Psi)$ for some map $\Psi: P \times P \rightarrow \operatorname{Id}\left(R_{R}\right)$ satisfying the conditions of (2).

Proof. Statements (1) and (2) are straightforward to verify, using the definition of multiplication in the incidence ring $A=I(P, R)$.

For statement (3), assume that $I$ is a two-sided ideal of $A$. For every $x \leq y$ in $P$ let $\Psi(x, y)=\{\alpha(x, y) \mid \alpha \in I\}$. Clearly $\Psi(x, y)$ is a two-sided ideal of $R$, and $I \subseteq I(\Psi)$. Let $\alpha \in I(\Psi)$. Then for every pair $x \leq y \in P$ there is $\alpha_{x, y} \in I$ such that $\alpha_{x, y}(x, y)=\alpha(x, y)$. By the finiteness of $P$ we may then write $\alpha=\sum_{x \leq y} e_{x} \alpha_{x, y} e_{y}$, which is an element of $I$. Therefore $I=I(\Psi)$ and hence $\Psi$ satisfies the conditions of (2).

Having associated the two-sided ideals of $A$ with certain maps from $P \times P$ to $\operatorname{Id}(R)$, we now determine additional properties of such maps which ensure that the associated ideal of $A$ is in fact an element of $D(A)$. Specifically, given any map $F: \hat{P} \rightarrow \operatorname{Id}\left(R_{R}\right)$ we define

$$
\begin{gathered}
\Psi_{F}: P \times P \rightarrow \operatorname{Id}\left(R_{R}\right) \text { by } \Psi_{F}(x, y)=F(\hat{x}), \quad \text { and } \\
J(F)=I\left(\Psi_{F}\right) .
\end{gathered}
$$

From the matrix point of view, $J(F)$ is the collection of matrices in $I(P, R)$ for which the entry in the $(x, y)$ coordinate is taken from the right ideal $F(\hat{x})$. In particular, the entries in a given row of an element of $J(F)$ belong to the fixed right ideal $F(\hat{x})$.

Lemma 1.2. Let $P$ be a preordered set. Let $F$ be a map from $\hat{P}$ to $\operatorname{Id}\left(R_{R}\right)$. Then:

(1) $J(F)$ is a right ideal of $A$.

(2) $J(F)$ is a two-sided ideal of $A$ if and only if $\operatorname{Im} F \subseteq \operatorname{Id}(R)$ and $F$ is an anti-homomorphism of partially ordered sets.

(3) If $P$ is finite, then $D(A) \simeq\{J(F) \mid F \in \operatorname{AntiHom}(\hat{P}, D(R))\}$.

Proof. (1) and (2) are consequences of Lemma 1.1. 
(3) Let $F \in \operatorname{AntiHom}(\hat{P}, D(R))$. For every $x \in \hat{P}$, let $F_{1}(x) \in \operatorname{Id}\left(R_{R}\right)$ be a complement of $F(x)$, so that $F(x) \oplus F_{1}(x)=R$. Then it is easy to check that $J(F) \oplus J\left(F_{1}\right)=A$, so that $J(F) \in D(A)$.

Conversely, let $J=f A \in D(A)$; so $f=f^{2} \in A$, and $A f \subseteq f A$. For every $X \in \hat{P}$ let $f_{X} \in M_{X}(R)$ be given by $f_{X}(x, y)=f(x, y)$ for every $x, y \in X$. If $x, y \in X, f_{X}^{2}(x, y)=\sum_{z \in X} f_{X}(x, z) f_{X}(z, y)=\sum_{x \leq z \leq y} f(x, z) f(z, y)=$ $f(x, y)=f_{X}(x, y)$. Thus $f_{X}$ is an idempotent of $M_{X}(R)$. Moreover $f_{X} M_{X}(R) \in D\left(M_{X}(R)\right)$ because if $r \in M_{X}(R)$, then there is $\alpha \in A$ such that $r e_{X} f=f \alpha$ and hence $r f_{X}=f_{X} \alpha_{X}$ (where $\alpha_{X}$ is defined similarly). Since the ideals of $M_{X}(R)$ each arise from an ideal of $R$, we have that there is a two-sided ideal $K_{X}$ of $R$ such that $f_{X} M_{X}(R)=M_{X}\left(K_{X}\right)$. But $M_{X}\left(K_{X}\right)$ is then also a direct summand of $M_{X}(R)_{M_{X}(R)}$, and hence $e_{x} M_{X}\left(K_{X}\right)$ is a direct summand of $e_{x} M_{X}(R)_{M_{X}(R)}$ for every $x \in X$. Since the functor given by $e_{x} M_{X}(-)$ is precisely the equivalence functor between $\operatorname{Mod}-M_{X}(R)$ and $M o d-R$, we conclude that $K_{X}$ is a direct summand of $R_{R}$. Thus $K_{X}=f_{X}^{1} R$ for some idempotent $f_{X}^{1}$ of $R$ such that $R f_{X}^{1} \subseteq f_{X}^{1} R$. Consequently we have $f_{X} M_{X}(R)=M_{X}\left(K_{X}\right)=M_{X}\left(f_{X}^{1} R\right)=g_{X} \cdot M_{X}(R)$, where $g_{X}$ is the scalar matrix in $M_{X}(R)$ with $f_{X}^{1}$ on the diagonal. In particular, $f_{X}$ generates the same right (in fact, two-sided) ideal of $M_{X}(R)$ as the scalar $g_{X}$.

Now let $F: \hat{P} \rightarrow D(R)$ be given by $F(X)=f_{X}^{1} R$. We define $f_{1} \in A$ by setting

$$
f_{1}(x, y)=f_{X}^{1} \text { if } \hat{x}=\hat{y}=X \text {, while } f_{1}(x, y)=0 \text { otherwise. }
$$

So $f_{1}$ is simply the diagonal blocks of $f$. As such, using the finiteness of $P$ we may write $f_{1}=\sum_{\{(x, y) \mid \hat{x}=\hat{y}\}} e_{x} f e_{y}$, so that $f_{1} \in f A$ as $f A$ is two-sided (being an element of $D(A)$ ). In particular, since $f$ is idempotent we get that $f f_{1}=f_{1}$. Again using the finiteness of $P$ we have that $f-f_{1}$ is nilpotent (necessarily of degree $\leq|P|)$. Expanding the equation $\left(f-f_{1}\right)^{t}=0$ and using that $f f_{1}=f_{1}$ yields that $f=f^{t} \in f_{1} A$. Thus we have that $f A=f_{1} A$. Now let $f_{2}$ be the block-diagonal element of $A$ whose entry in the $X-X$ component is $g_{X}$. By the discussion in the previous paragraph we have that $f_{1} A=f_{2} A$, so that $f A=f_{2} A$. But the diagonal form of $f_{2}$ immediately yields that $f_{2} A=J(F)$, so that finally we have shown that $J=f A=$ $f_{2} A=J(F)$ is of the required form. That $F \in \operatorname{AntiHom}(\hat{P}, D(R))$ follows from part (2).

Definition 1.3. Let $P$ be a partially ordered set. We define the partially ordered subset $P_{1}$ of $P$ by setting

$P_{1}=\left\{x \in P \mid\right.$ there exists $x^{\prime}<x$ with the property that

$$
\left.y \leq x^{\prime} \text { for every } y<x\right\} .
$$

In other words, $x$ is in $P_{1}$ in case there exists a unique maximal element $x^{\prime}$ in $X$ below $x$. 
If $T$ is any ring then as noted above $D(T)$ is a partially ordered set under inclusion. In the sequel we will focus much attention on the partially ordered subset $D(T)_{1}$ of $D(T)$. We first prove a general result about such subsets.

Lemma 1.4. Let $P$ and $Q$ be two partially ordered sets, such that $P$ is finite, and such that $Q$ has a unique minimal element 0 . Let $H: P \times Q \rightarrow$ $\operatorname{AntiHom}(P, Q)$ be the map given by setting $H(p, q)(x)=q$ if $x \leq p$ and $H(p, q)(x)=0$ otherwise. Then $H$ restricts to an isomorphism of partially ordered sets

$$
H: P \times Q_{1} \simeq \operatorname{AntiHom}(P, Q)_{1} .
$$

In particular, for every $(p, q) \in P \times Q_{1}$, the unique maximal element $H(p, q)^{\prime}$ $<H(p, q)$ is given by setting $H(p, q)^{\prime}(x)=q$ if $x<p, H(p, q)^{\prime}(p)=q^{\prime}$, and $H(p, q)^{\prime}(x)=0$ otherwise.

Proof. First we check that $H=H(p, q) \in \operatorname{AntiHom}(P, Q)$. Indeed, suppose $x \leq y$ in $P$. Then either $y \leq p$, in which case $H(y)=q=H(x)$, or $y \not \leq p$, in which case $H(y)=0 \leq H(x)$.

It is clear that the restriction of $H$ to $P \times(Q \backslash\{0\})$ is injective and preserves the order. So we must check that $H\left(P \times Q_{1}\right)=\operatorname{AntiHom}(P, Q)_{1}$, and that the inverse map preserves the order. To this end, suppose that $(p, q) \in P \times Q_{1}$. Let $H^{\prime}: P \rightarrow Q$ be given by $H^{\prime}(x)=q$ if $x<p, H^{\prime}(p)=q^{\prime}$, and $H^{\prime}(x)=0$ if $x \not \leq p$. Then $H^{\prime}<H=H(p, q)$. We verify that $H^{\prime}$ satisfies the desired properties. So let $G<H$ in $\operatorname{AntiHom}(P, Q)$; we show $G \leq H^{\prime}$. Now $G(x) \leq H(x)=0$ if $x \not \leq p$ and hence $G(x)=0$ in this case. Moreover, $G(p) \leq H(p)=q$. Assume that $G(p)=q$. If $x \leq p$, then $q=$ $G(p) \leq G(x) \leq H(p)=q$; but this gives $G=H$, a contradiction. Therefore $G(p)<q$, and hence $G(p) \leq q^{\prime}$. Finally, if $x<p$, then $G(x) \leq H(x)=q$. Thus $G \leq H^{\prime}$ and this proves that $H \in \operatorname{AntiHom}(P, Q)_{1}$.

Conversely, let $H \in \operatorname{AntiHom}(P, Q)_{1}$. Let $p$ and $p_{1}$ be two different maximal elements of $\{x \in P: H(x) \neq 0\}$. Let $F_{1}, F_{2}: P \rightarrow Q$ be given by

$$
\begin{gathered}
F_{1}(x)=F_{2}(x)=H(x) \text { for } p \neq x \neq p_{1} \text { and } \\
F_{1}(p)=H(p), \quad F_{1}\left(p_{1}\right)=0, \quad F_{2}(p)=0, \quad F_{2}\left(p_{1}\right)=H\left(p_{1}\right) .
\end{gathered}
$$

Since $F_{1}<H$ and $F_{2}<H$ we have $F_{1} \leq H^{\prime}$ and $F_{2} \leq H^{\prime}$. Then $H(x)=$ $F_{1}(x) \leq H^{\prime}(x) \geq F_{2}(x)=H(x)$ for $p \neq x \neq p_{1}, H(p)=F_{1}(p) \leq H^{\prime}(p) \leq$ $H(p)$, and $H\left(p^{\prime}\right)=F_{2}\left(p^{\prime}\right) \leq H^{\prime}\left(p^{\prime}\right) \leq H\left(p^{\prime}\right)$. But this gives $H=H^{\prime}$, a contradiction. Therefore there is an element $p \in P$, such that $H(p) \neq 0$ and $H(x)=0$, for every $x \not \leq p$.

Let $F: P \rightarrow Q$ be given by $F(x)=H(x)$ if $x \neq p$ and $F(p)=0$. Since $F \in \operatorname{AntiHom}(P, Q)$ and $F<H$, then $F \leq H^{\prime}$. Therefore $H(x) \geq H^{\prime}(x) \geq$ $F(x)=H(x)$, and hence $H(x)=H^{\prime}(x)$, for every $x \neq p$. Since $H^{\prime}<H$, then $H^{\prime}(p)<H(p)$.

Set $q=H(p)$. If $H \neq H(p, q)$, there is $x<p$ such that $H(x)>q$. Assume that $x$ is maximal with that condition. Let $F \in \operatorname{AntiHom}(P, Q)$ be given 
by $F(y)=H(y)$ if $y \neq x$, and $F(x)=q$. Then $F<H$ and hence $F \leq H^{\prime}$. Therefore $q=F(p) \leq H^{\prime}(p) \leq H(p)=q$. Thus $H^{\prime}=H$ a contradiction. We conclude that $H=H(p, q)$.

We now show that $q \in Q_{1}$. We prove that $H^{\prime}(p)$ has the desired properties; i.e., we prove that $H^{\prime}(p)=q^{\prime}$. If $x<q$, let $F \in \operatorname{AntiHom}(P, Q)$ be given by $F(y)=H(y)$ if $y \neq p$ and $F(p)=x$. Since $F<H$, then $F \leq H^{\prime}$ and hence $x \leq H^{\prime}(p)$.

Now that we have shown that $H$ is a set bijection which preserves the order, we need only show that $H^{-1}$ preserves the order as well. So suppose that $H\left(p_{1}, q_{1}\right) \leq H\left(p_{2}, q_{2}\right)$. Then on applying each of these antihomomorphisms to $p_{1} \in P$ we get $q_{1}=H\left(p_{1}, q_{1}\right)\left(p_{1}\right) \leq H\left(p_{2}, q_{2}\right)\left(p_{1}\right)$. But $q_{1} \neq 0$, so $H\left(p_{2}, q_{2}\right)\left(p_{1}\right) \neq 0$, which by definition of $H\left(p_{2}, q_{2}\right)$ gives that $p_{1} \leq p_{2}$, and that $q_{1} \leq q_{2}$.

Lemmas 1.2 and 1.4 now yield:

Proposition 1.5. Let $P$ be a finite preordered set, and let $R$ be any ring. Then there is an isomorphism of partially ordered sets

$$
H: \hat{P} \times D(R)_{1} \simeq D(A)_{1} .
$$

Specifically, for $(X, K) \in \hat{P} \times D(R)_{1}$ we set $H(X, K)=J\left(F_{X, K}\right)$, where $F_{X, K} \in \operatorname{AntiHom}\left(\hat{P}, D(R)_{1}\right)$ is defined by setting $F_{X, K}(W)=K$ if $W \leq X, F_{X, K}(W)=0$ otherwise .

In turn, Proposition 1.5 allows us to establish one of the two Solutions to the Isomorphism Problem mentioned in the introduction.

Theorem 1.6. Let $P$ and $P^{\prime}$ be finite partially ordered sets, and let $R$ be a ring for which $D(R)$ is finite. If the incidence rings $I(P, R)$ and $I\left(P^{\prime}, R\right)$ are isomorphic, then $P$ and $P^{\prime}$ are isomorphic as partially ordered sets.

Proof. As $P$ and $P^{\prime}$ are partially ordered we have $P=\hat{P}$ and $P^{\prime}=\widehat{P^{\prime}}$. Let $A$ denote $I(P, R)$ and let $A^{\prime}$ denote $I\left(P^{\prime}, R\right)$. It is straightforward to show that a ring isomorphism between $A$ and $A^{\prime}$ yields an isomorphism of the corresponding partially ordered sets $D(A)_{1}$ and $D\left(A^{\prime}\right)_{1}$. By Lemma 1.5 this yields an isomorphism of partially ordered sets $P \times D(R)_{1} \simeq P^{\prime} \times D(R)_{1}$. Now an application of [2, Theorem 4.3$]$ yields the desired isomorphism between $P$ and $P^{\prime}$.

As is apparent from the proof of Lemma 1.2, $D(T) \simeq D\left(M_{n}(T)\right)$ for any ring $T$ and any matrix ring $M_{n}(T)$. Thus, as $M_{n}(T)=I(\hat{x}, T)$ for $|\hat{x}|=n, D(-)$ cannot be used to recover preordered sets. Therefore, in order to establish a result similar to that given in Theorem 1.6 in the more general context of preordered sets, we must utilize the more subtle structure $D^{*}(-)$ (defined below). The price we pay for weakening the hypothesis on 
the ordered sets is the necessity of imposing additional structure on the underlying coefficient ring.

Definition 1.7. For any ring $T$ and any right $T$-module $M_{T}$ we define the summand length of $M$, denoted $w\left(M_{T}\right)$, by setting

$$
w\left(M_{T}\right)=\max \left\{n \mid M=\oplus_{i=1}^{n} M_{i} \text {, for some nonzero submodules } M_{i} \leq M\right\} .
$$

We set $w\left(M_{T}\right)=\infty$ if no such maximum exists. We say the $\operatorname{ring} T$ has finite summand length in case $w\left(T_{T}\right)$ is finite.

We note that the definition of finite summand length for a ring $T$ given above is left/right symmetric. To see this, we observe that any direct decomposition of the right regular module $T_{T}$ having $n$ nonzero summands gives rise to a complete set of $n$ orthogonal idempotents in $T$. This set of idempotents can then be used to give a direct decomposition of the left regular module ${ }_{T} T$ having $n$ nonzero direct summands. The desired conclusion follows by symmetry.

Lemma 1.8. For any ring $T$, if $T$ has finite summand length, then $D(T)$ is finite.

Proof. We denote $w\left(T_{T}\right)$ by $N$. Since any chain of proper inclusions of length $n$ of nonzero elements of $D(T)$ gives rise to a decomposition of $T_{T}$ containing $n+1$ summands, we conclude that the partially ordered set $D(T)$ has finite height (in fact, height $\leq N$ ). Thus to show that $D(T)$ is finite it is enough to show that for every $K \in D(T)$, the set of minimal elements of $\{J \in D(T) \mid K \subset J\}$ is finite. Indeed, if we denote this set of minimal elements by $\left\{J_{i} \mid i \in \Omega\right\}$, then we show that this set contains at most $N$ elements, by showing that this set can be used to generate an equal-sized set of nonzero pairwise orthogonal idempotents in $T$. Let $K=g T, J_{i}=e_{i} T$ for idempotents $g,\left\{e_{i}\right\}_{i \in \Omega}$. Since $K \subset J_{i}$ we have $e_{i} g=g$ for all $i$. With this it is easy to show that each $e_{i}^{\prime}=e_{i}-g e_{i}$ is idempotent. Further, it is easy to show that $e_{i} T \cap e_{j} T=e_{i} e_{j} T \in D(T)$; the minimality property of these ideals then implies that $e_{i} e_{j} T=g T$ for $i \neq j$. It follows that $e_{i}^{\prime}$ and $e_{j}^{\prime}$ are in fact orthogonal for $i \neq j$. Since $e_{i} \neq g$ we have that $e_{i}^{\prime}$ is nonzero for all $i$, and so $\left\{e_{i}^{\prime}\right\}_{i \in \Omega}$ is a set of nonzero pairwise orthogonal idempotents. But a set of more than $N$ nonzero pairwise orthogonal idempotents in $T$ would yield a decomposition of $T_{T}$ in violation of the hypothesis that $w\left(T_{T}\right)=N$, so that $|\Omega| \leq N$ as desired.

Definition 1.9. Let $T$ be any ring with finite summand length. We define

$$
D^{*}(T)=\left\{(K, i) \mid K \in D(T)_{1} \text { and } 1 \leq i \leq w\left(\left(K / K^{\prime}\right)_{T}\right)\right\} .
$$

(Here $K^{\prime}$ is the unique maximal element of $D(T)$ below $K$, as given in Definition 1.3.) We note that $w\left(\left(K / K^{\prime}\right)_{T}\right)$ is indeed an integer, as $K / K^{\prime}$ is 
isomorphic to a direct summand of $T_{T}$. We define a preorder on $D^{*}(T)$ by setting

$$
(K, i) \leq(L, j) \Leftrightarrow K \subseteq L .
$$

For any positive integer $s$, let $\mathbf{N}_{s}$ denote the set $\{1,2, \ldots, s\}$.

Lemma 1.10. Let $P$ be a finite preordered set, and let $R$ be a ring with finite summand length. If $K \in D(R)_{1}$ and $X \in \hat{P}$, then

$$
|X| \cdot w\left(K / K_{R}^{\prime}\right)=w\left(H(X, K) / H(X, K)_{A}^{\prime}\right) .
$$

In particular, there exists a bijection

$$
\phi_{X, K}: X \times \mathbf{N}_{w\left(K / K^{\prime}\right)} \rightarrow \mathbf{N}_{w\left(H(X, K) / H(X, K)^{\prime}\right)} .
$$

Proof. Let $h(X, K)$ denote the right ideal $e_{X} H(X, K)$ of $A$. By Lemma 1.4 one can show that $H(X, K)=\left(\sum_{Y<X} H(Y, K)\right) \oplus h(X, K)$, and that the unique maximal element $H(X, K)^{\prime}$ below $H(X, K)$ is given by $H(X, K)^{\prime}=$ $\left(\sum_{Y<X} H(Y, K)\right) \oplus h\left(X, K^{\prime}\right)$. $h(X, K) / h\left(X, K^{\prime}\right)$.

Now let $J=I\left(P, K^{\prime}\right)$. Since $h(X, K) J \subset h\left(X, K^{\prime}\right)$, then $h(X, K) / h\left(X, K^{\prime}\right)$ is a right $A / J$-module. Moreover, if $B=I\left(P, R / K^{\prime}\right)$, then $\left(h(X, K) / h\left(X, K^{\prime}\right)\right)_{A / J} \simeq h\left(X, K / K^{\prime}\right)_{B}$ and it follows that $w\left(\left(h(X, K) / h\left(X, K^{\prime}\right)\right)_{A}\right)=w\left(\left(h(X, K) / h\left(X, K^{\prime}\right)\right)_{A / J}\right)=w\left(h\left(X, K / K^{\prime}\right)_{B}\right)$. Consequently, $w\left(H(X, K) / H(X, K)_{A}^{\prime}\right)=w\left(h\left(X, K / K^{\prime}\right)_{B}\right)$. Since $K / K^{\prime}$ is isomorphic to a direct summand of $K$ it is in fact isomorphic to a right ideal of $R$; thus it suffices to prove that if $L$ is a right ideal of $R$, then $w\left(h(X, L)_{A}\right)=|X| w\left(L_{R}\right)$.

If $L=\oplus_{i=1}^{n} L_{i}$, then $h(X, L)=\oplus_{x \in X} \oplus_{i=1}^{n} e_{x} h\left(X, L_{i}\right)$ since $e_{X}$ acts like an identity on the left of elements of $h(X, L)$. This shows that $w(h(X, L)) \geq$ $|X| w(L)$. Now suppose $w(h(X, L))=n$ and so we may write $h(X, L)=$ $\oplus_{i=1}^{n} L_{i}$ where each $L_{i}$ is a nonzero right ideal of $A$. Again, since $e_{X}$ is an identity on the left of each $L_{i}$, we have $L_{i}=\oplus_{x \in X} e_{x} L_{i}$ and so $h(X, L)=$ $\oplus_{i=1}^{n} \oplus_{x \in X} e_{x} L_{i}$. Since $n=w(h(X, L))$, it follows that, for every $i=1, \ldots, n$, there is exactly one $x_{i} \in X$ such that $L_{i}=e_{x_{i}} L_{i}$. (It may be the case that two such $L_{i}$ share the same $x$.) For every $i=1, \ldots, n$, let $J_{i}=\left\{\alpha\left(x_{i}, x_{i}\right) \mid\right.$ $\left.\alpha \in L_{i}\right\}$. It is clear that each $J_{i}$ is a right ideal of $R$ and that, for every $x \in X, L=\sum_{i, x_{i}=x} J_{i}$. We claim that this sum is direct. To see this, suppose $\sum_{i, x_{i}=x} r_{i}=0$, with $r_{i} \in J_{i}$, and so there are $\alpha_{i} \in L_{i}$ such that $\alpha_{i}(x, x)=r_{i}$. Since $\alpha_{i} \in L_{i}=e_{x_{i}} L_{i}$, it follows that $\sum_{i=1}^{n} \alpha_{i} e_{x}=0$. Since the original sum $\oplus_{i=1}^{n} L_{i}$ is direct, we have each $\alpha_{i} e_{x}=0$ and so each $r_{i}=0$ and the claim holds. In particular, the number $m_{x}$ of the $i, i=1, \ldots, n$ such that $x_{i}=x$ satisfies $m_{x} \leq w(L)$. Consequently, $h(X, L)=\oplus_{x \in X} e_{x} h(X, L)=$ $\oplus_{x \in X} \oplus_{i, x_{i}=x} e_{x} h\left(X, J_{i}\right)$. Once we show that $e_{x_{i}} h\left(X, J_{i}\right)=L_{i}$ for every $i$, the proof will be complete because then we shall have $w(h(X, L))=$ 
$\sum_{x \in X} m_{x} \leq|X| w(L)$, as desired. To show the equality, let $\alpha \in e_{x_{i}} h\left(X, J_{i}\right)$. Then, for $y \geq x_{i}$, there are $\beta_{y} \in L_{i}$ such that $\alpha\left(x_{i}, y\right)=\beta_{y}\left(x_{i}, x_{i}\right)$ and so $\alpha=\sum_{y \geq x} \beta_{y} e_{x_{i}, y} \in L_{i}$ which shows that $e_{x_{i}} h\left(X, J_{i}\right) \subset L_{i}$. However, since $e_{x_{i}} h\left(X, J_{i}\right)$ and $L_{i}$ are summands of $h(X, L)$, we see from the Modular Law that $L_{i}=e_{x_{i}} h\left(X, J_{i}\right)$.

Proposition 1.11. If $P$ is any finite preordered set, and $R$ is a ring with finite summand length, then $P \times D^{*}(R) \simeq D^{*}(A)$.

Proof. Let $H$ denote the isomorphism given in Proposition 1.5. For every $X \in \hat{P}$ and $K \in D(R)_{1}$, let $\phi_{X, K}: X \times \mathbf{N}_{w\left(K_{R}\right)} \rightarrow \mathbf{N}_{w\left(h(X, K)_{A}\right)}$ be the map described in Lemma 1.10. We now define $\Phi: P \times D^{*}(R) \rightarrow D^{*}(A)$ by setting

$$
\Phi(x,(K, i))=\left(H(\hat{x}, K), \phi_{\hat{x}, K}(x, i)\right) .
$$

Using the fact that $H$ is an isomorphism of partially ordered sets and that $\phi_{X, K}$ is a bijection, it is straightforward to check that $\Phi$ is indeed an isomorphism of preordered sets.

We now have all the tools required to establish our second version of the Isomorphism Problem.

Theorem 1.12. Let $P$ and $P^{\prime}$ be two finite preordered sets, and let $R$ be a ring with finite summand length. If $I(P, R) \simeq I\left(P^{\prime}, R\right)$ as rings, then $P \simeq P^{\prime}$ as preordered sets.

Proof. Let $A$ denote $I(P, R)$ and let $A^{\prime}$ denote $I\left(P^{\prime}, R\right)$. It is straightforward to show that a ring isomorphism between $A$ and $A^{\prime}$ yields an isomorphism of the corresponding preordered sets $D^{*}(A)$ and $D^{*}\left(A^{\prime}\right)$. By Proposition 1.11, this gives an isomorphism of preordered sets

$$
P \times D^{*}(R) \simeq P^{\prime} \times D^{*}(R) .
$$

Now an application of [2, Theorem 4.3$]$ yields the desired isomorphism between $P$ and $P^{\prime}$.

We note that Theorem 1.12 answers the question posed at the end of [1]; specifically, we have shown that the Isomorphism Problem has a positive solution when the preordered sets are finite, and the coefficient ring is right or left noetherian.

\section{Non-extendability of Solutions.}

In the second section of this article we present three constructions which demonstrate the failure of possible extensions of various solutions of the Isomorphism Problem.

Our first non-extendability result shows that the hypotheses of Theorem 1.12 cannot be replaced by the weaker hypotheses of Theorem 1.6. 
Proposition 2.1. There exists a ring $S$ having finite $D(S)$ and there exist nonisomorphic preordered sets $P$ and $P^{\prime}$ such that $I(P, S) \simeq I\left(P^{\prime}, S\right)$.

Proof. Let $k$ be any unital ring, and let $S$ denote the $\operatorname{ring} R F M(k)$ of countably infinite row-finite matrices over $k$. It is well-known that $S$ has the property that $S \simeq M_{2}(S)$, which yields that $I(P, S) \simeq I\left(P^{\prime}, S\right)$ where $|P|=1$ and $P^{\prime}=\hat{x}$ where $|\hat{x}|=2$. Furthermore, since $S$ has only one nontrivial two-sided ideal, $D(S)$ is finite.

Prior to presenting our second non-extendability result (Proposition 2.3 below), we review some of the ideas introduced in [4]. Let $X$ be any locally finite preordered set, and let $a \in X$. We define

$$
\hat{X}^{a}=\left\{\left(x_{n}\right) \in \prod_{n \in \mathbf{N}} X \mid \exists N \text { with } x_{n}=a \forall n \geq N\right\} .
$$

In [4, Proposition 1] Voss shows that $\hat{X}^{a}$ is a locally finite preordered set having the property that $X \times \hat{X}^{a} \simeq \hat{X}^{a}$ as preordered sets. Indeed, the isomorphism $\phi$ is given by setting $\phi\left(x,\left(x_{n}\right)\right)=\left(y_{n}\right)$, where $y_{1}=x$ and $y_{n}=x_{n-1}$ for all $n \geq 2$.

We now let $\left\{X_{i}\right\}_{i \in \Omega}$ be any collection of preordered sets. For each $i \in \Omega$ let $a_{i}$ denote a fixed element of $X_{i}$, and let $\bar{a}_{i}$ denote the element $\left(a_{i}, a_{i}, \ldots\right)$ of $\hat{X}_{i}{ }^{a_{i}}$. Finally, let $B$ denote the set

$$
B=\left\{\left(b_{i}\right) \in \prod_{i \in \Omega} \hat{X}_{i}^{a_{i}} \mid b_{i}=\bar{a}_{i} \text { for all but finitely many } i\right\} .
$$

Lemma 2.2. The preordered set $B$ defined above is locally finite provided that each $X_{i}$ is locally finite. Moreover, for each $i \in \Omega$ we have $X_{i} \times B \cong B$ as preordered sets.

Proof. The set $B$ inherits the preorder from the product $\prod_{i \in \Omega} \hat{X}_{i}{ }^{a_{i}}$. Now choose $\left(b_{i}\right) \leq\left(c_{i}\right) \leq\left(d_{i}\right)$ in $B$. Then for all but finitely many $i \in \Omega$ we have that $b_{i}=c_{i}=d_{i}=\bar{a}_{i}$. The first statement now follows as each $\hat{X}_{i}{ }^{a_{i}}$ is locally finite.

Define $\phi_{i}: X_{i} \times B \rightarrow B$ by setting $\phi_{i}\left(\left(x_{i},\left(b_{j}\right)\right)\right)=\left(c_{j}\right)$, where $c_{j}=b_{j}$ in $\hat{X}_{j}{ }^{a_{j}}$ for $j \neq i$, and $c_{i}=\phi\left(\left(x_{i}, b_{i}\right)\right.$ ) (using the map $\phi$ described above). Then it is easy to check that $\phi_{i}$ is an isomorphism of the desired type.

Our second non-extendability result shows that the Recovery Question must always fail without some stipulation on the structure of the underlying coefficient rings.

Proposition 2.3. Let $\left\{X_{i}\right\}_{i \in \Omega}$ be any collection of locally finite preordered sets. Then there exists a ring $S$ with the property that the incidence rings 
$I\left(X_{i}, S\right)$ and $I\left(X_{j}, S\right)$ are isomorphic for all $i, j \in \Omega$. Indeed, each of these incidence rings is isomorphic to $S$ itself.

Proof. Let $B$ be the locally finite preordered set defined above, let $T$ be any ring, and let $S$ denote the incidence ring $I(B, T)$. Then for each $i \in \Omega$,

$$
I\left(X_{i}, S\right) \cong I\left(X_{i}, I(B, T)\right) \cong I\left(X_{i} \times B, T\right) \cong I(B, T) \cong S
$$

We mention that the above result generalizes an example from [1]. In [1, Example 1.1] Dăscălescu and Van Wyk construct a ring $R$ for which the following five 'structural matrix rings over $R$ ' are isomorphic:

$$
R \quad R \oplus R \quad M_{2}(R) \quad\left(\begin{array}{cc}
R & R \\
0 & R
\end{array}\right) \quad\left(\begin{array}{cc}
R & 0 \\
R & R
\end{array}\right) .
$$

These structural matrix rings are precisely the incidence rings which arise from the five preordered sets (respectively)

$$
\{a\} \quad\{a, b\} \quad\{a, b \mid a \leq b, b \leq a\} \quad\{a, b \mid a \leq b\} \quad\{a, b \mid b \leq a\} .
$$

The final non-extendability result (Proposition 2.5) addresses the necessity of the finiteness condition on the preordered sets. The key ingredient in this result is an example of non-cancellability for certain preordered sets. In [4, Theorem 4] and a subsequent remark, Voss proves that if $X, Y$ and $Z$ are preordered sets for which $X$ and $Y$ are locally finite, and $Z$ is finite and has the property that the connected components of $Z$ are pairwise isomorphic, then $X \times Z \simeq Y \times Z$ implies $X \simeq Y$. We now show that the condition on the connected components of $Z$ cannot be dropped from the hypotheses of Voss' result. We recall some notation: For $x, y$ in a preordered set $X$ we say that $y$ covers $x$ in case $x<y$, and there does not exist $z$ in $X$ with $x<z<y$.

Proposition 2.4. There exists a finite (necessarily non-connected) partially ordered set $Z$ and locally finite (necessarily infinite) partially ordered sets $X$ and $Y$ such that $X \times Z \simeq Y \times Z$ but $X \not Y$.

Proof. Let $U$ be any preordered set in which there exists a unique minimal element $a \in U$. Let $\hat{U}^{a}$ be the preordered set constructed as above, and let $\bar{a}$ denote the constant tuple $\bar{a}=(a, a, \ldots)$. Set

$$
\begin{aligned}
& \hat{U}_{0}^{a}=\left\{\left(a_{n}\right) \in \hat{U}^{a} \mid \max \left\{n \mid a_{n} \neq a\right\} \text { is even }\right\} \cup\{\bar{a}\} \quad \text { and } \\
& \hat{U}_{1}^{a}=\left\{\left(a_{n}\right) \in \hat{U}^{a} \mid \max \left\{n \mid a_{n} \neq a\right\} \text { is odd }\right\} \cup\{\bar{a}\} .
\end{aligned}
$$

We first show that $U \times \hat{U}_{0}^{a} \simeq \hat{U}_{1}^{a}$ and $U \times \hat{U}_{1}^{a} \simeq \hat{U}_{0}^{a}$ as preordered sets. To verify this, it suffices to prove $U \times \hat{U}_{0}^{a} \simeq \hat{U}_{1}^{a}$, since the other isomorphism is 
shown symmetrically. Define $\phi: U \times \hat{U}_{0}^{a} \rightarrow \hat{U}_{1}^{a}$ by setting $\phi(x, f)(1)=x$ and $\phi(x, f)(n)=f(n-1)$ for $n \geq 2$. That $\phi$ is a set bijection which preserves the underlying preordering is clear.

We next show, perhaps counter to one's intuition, that the preordered sets $\hat{U}_{0}^{a}$ and $\hat{U}_{1}^{a}$ are not isomorphic, as long as there exists a unique cover $\alpha$ of $a$ in $U$.

Clearly $\bar{a}$ is the unique minimal element of both $\hat{U}_{0}^{a}$ and $\hat{U}_{1}^{a}$. Let $C_{0}$ denote the set of covers of $\bar{a}$ in $\hat{U}_{0}^{a}$; so the elements of $C_{0}$ are of the form $\widehat{\alpha_{2 n}}$, the tuple whose $2 n$-th coordinate is $\alpha$, and all other coordinates equal a. Similarly, the set $C_{1}$ of covers of $\bar{a}$ in $\hat{U}_{1}^{a}$ are of the form $\widehat{\alpha_{2 n+1}}$.

It is easy to see that every element of $C_{0}$ is covered by an element which does not cover any other element of $C_{0}$; for instance, $\widehat{\alpha_{2 n}}$ is covered in such a way by $\widehat{d_{2 n}}$, the tuple which is $\alpha$ in coordinates 1 and $2 n$, and is $a$ elsewhere. However, the element $\widehat{\alpha_{1}}$ of $C_{1}$ does not have this property; that is, every cover of $\widehat{\alpha_{1}}$ necessarily covers another element of $C_{1}$.

Since $\bar{a}$ serves as the unique minimal element for both $\hat{U}_{0}^{a}$ and $\hat{U}_{1}^{a}$, and since covers are preserved by preordered set isomorphism, the above discussion yields that $\hat{U}_{0}^{a} \not \hat{U}_{1}^{a}$ as claimed.

Now we construct the example in which cancellation fails. Let $U$ and $V$ be two preordered sets, with elements $a \in U$ and $b \in V$. Set $Z=U \uplus V$ (the disjoint union), $X=\hat{U}_{1}^{a} \times \hat{V}_{0}^{b}$, and $Y=\hat{U}_{0}^{a} \times \hat{V}_{1}^{b}$. Then

$$
\begin{aligned}
X \times Z & \simeq(U \uplus V) \times\left(\hat{U}_{1}^{a} \times \hat{V}_{0}^{b}\right) \simeq\left(U \times \hat{U}_{1}^{a} \times \hat{V}_{0}^{b}\right) \uplus\left(V \times \hat{U}_{1}^{a} \times \hat{V}_{0}^{b}\right) \\
& \simeq\left(\hat{U}_{0}^{a} \times V \times \hat{V}_{1}^{b}\right) \uplus\left(U \times \hat{U}_{0}^{a} \times V_{1}^{b}\right) \\
& \simeq(U \uplus V) \times\left(\hat{U}_{0}^{a} \times \hat{V}_{1}^{b}\right)=Y \times Z .
\end{aligned}
$$

Consequently, to find an example of the desired type, it suffices to find $U$ and $V$ such that $X=\hat{U}_{0}^{a} \times \hat{V}_{1}^{b} \not \hat{U}_{1}^{a} \times \hat{V}_{0}^{b}=Y$. But if $U=\{a, \alpha\}$ such that $a<\alpha$ and $V=\{b\}$, then $\hat{V}_{0}^{b}=\{\bar{b}\}=\hat{V}_{1}^{b}$, while $\hat{U}_{0}^{a} \not \hat{U}_{1}^{a}$ as shown above. Then $X \simeq \hat{U}_{1}^{a} \not \hat{U}_{0}^{a} \simeq Y$, and we are done.

Our final non-extendability result shows that if we allow the preordered sets to be infinite, then we need not be able to recover the preordered sets, even from incidence rings with coefficients taken from a finite dimensional algebra.

Proposition 2.5. There exist locally finite, non-isomorphic partially ordered sets $P$ and $P^{\prime}$ and a finite dimensional algebra $R$ such that the incidence rings $I(P, R)$ and $I\left(P^{\prime}, R\right)$ are isomorphic.

Proof. Let $X, Y$ and $Z$ be as in Proposition 2.4 and let $k$ be any field. Let $P=X$, let $P^{\prime}=Y$, and let $R$ denote the incidence ring $I(Z, k)$. In particular, $R$ is a 4-dimensional algebra over $k$. By hypothesis we have 
$P \not P^{\prime}$. But we do have $P \times Z \simeq P^{\prime} \times Z$, and so

$$
\begin{aligned}
I(P, R) & =I(P, I(Z, k)) \simeq I(P \times Z, k) \simeq I\left(P^{\prime} \times Z, k\right) \\
& \simeq I\left(P^{\prime}, I(Z, k)\right)=I\left(P^{\prime}, R\right) .
\end{aligned}
$$

Since the ring $R$ given in Proposition 2.5 has finite $w(R)$ (and therefore also finite $D(R)$ ), we cannot extend either Theorem 1.6 or Theorem 1.12 to locally finite partially ordered sets.

At the end of [4], Voss asks whether or not his solution to the Isomorphism Problem (where the coefficient rings are assumed to be finite direct sums of indecomposable semiperfect rings each of whose associated preordered sets is pairwise isomorphic) extends to all artinian rings. Proposition 2.5 provides a negative answer to this question. In fact, the coefficient ring used in the Proposition is in some sense a minimal counterexample, as any algebra of dimension three or less over any field can be shown to satisfy Voss' hypotheses.

\section{References}

[1] S. Dăscălescu and L. Van Wyk, Do isomorphic structural matrix rings have isomorphic graphs?, Proc. A.M.S., 124(5) (1996), 1385-1391.

[2] L. Lovász, Operations with structures, Acta Mathematica Acad. Sci. Hung., 124(3-4) (1967), 321-328.

[3] E. Spiegel and C. O'Donnell, Incidence Algebras, Series in Pure and Applied Mathematics, Vol. 206, Marcel Dekker, New York, 1997.

[4] E.R. Voss, On the isomorphism problem for incidence rings, Illinois J. Math., 24 (1980), 624-638.

Received September 12, 1997. This work was completed while the third author was in residence at the University of Colorado at Colorado Springs, to which he wishes to express his thanks for its hospitality. The second author was supported by NSA grant MDA90496-0-0030, while the third author was supported by grants DGICYT (PB93-0515-C02-02) and DGICYES (PR95-469).

UNIVERSITY OF COLORADO

Colorado Springs, CO 80933

E-mail address: abrams@math.uccs.edu

University of Colorado

Colorado Springs, CO 80933

E-mail address: haefner@math.uccs.edu

UniversidAD DE MurCia

30071 MURCIA

SPAIN

E-mail address: adelrio@fcu.um.es 